\title{
Modelo transaccional de lectura para el desarrollo del modelo situacional de comprensión lectora, en los estudiantes de derecho
}

\section{Transactional reading model for the development of the situational model of reading comprehension in students}

\author{
Jaime Kuno Huayhua \\ jaimeskuno@hotmail.com \\ Universidad Mayor Real Y Pontificia de San Francisco Xavier de Chuquisaca, Bolivia
}

\section{RESUMEN}

Los modelos de lectura son creencias o conceptualizaciones que tiene el lector acerca de la lectura. En ella tiene que ver la formación recibida desde sus primeros cursos de formación escolar, muchas veces ha sido una formación receptiva y otras veces constructiva. El propósito de esta investigación fue determinar la influencia de los modelos de lectura en la utilización de estrategias de comprensión lectora, en los estudiantes de la Asignatura de Filosofía del Derecho. La metodología utilizada es cuantitativa no experimental, transeccional correlacional además se usó el método empírico para la medición. Se ha identificado dentro la influencia de los modelos de lectura en la utilización de estrategias de comprensión lectora en los estudiantes de la Asignatura de Filosofía del Derecho, cuatro componentes: El lector, el texto, el contexto, y la actividad. La relación teórica y gráfica de los cuatro componentes permitió fundamentar su relación interna y fundamentar de manera científica su proceso. Se obtuvo validación teórica y práctica de la propuesta pedagógica, obteniendo como resultado como adecuado las estrategias de comprensión lectora en el estudio.

Palabras clave: Modelos de lectura; comprensión lectora; estrategias de comprensión lectora

\section{ABSTRACT}

Reading models are beliefs or conceptualizations that the reader has about reading. It has to do with the training received since its first school training courses, many times it has been a receptive and sometimes constructive training. The purpose of this research was to determine the influence of reading models in the use of reading comprehension strategies in students of the Law Philosophy Subject. The methodology used is quantitative non-experimental, correlational translational and the empirical method was used for the measurement. The influence of reading models on the use of reading comprehension strategies has been identified within the students of the Law Philosophy Subject, four components: The reader, the text, the context, and the activity. The theoretical and graphic relationship of the four components allowed to base their internal relationship and to scientifically base their process. Theoretical and practical validation of the pedagogical proposal was obtained, obtaining as a result the reading comprehension strategies in the study as appropriate.

Key words: Reading models; reading comprehension; reading comprehension strategies 


\section{INTRODUCCIÓN}

La comprensión lectora, es uno de los procesos más complejos que desarrolla el ser humano para hacer suyo toda la cultura que le precede. Un estudio publicado en el periódico "La Razón", muestra cifras alarmantes con relación al desarrollo de las capacidades de comprensión lectora, en el que se da a conocer que en promedio, 56 de cada 100 estudiantes de sexto de primaria, del subsistema de educación regular, no son capaces de comprender lo que leen.

Este problema continúa siendo un factor negativo dentro de las esferas del subsistema de educación superior de formación profesional. Los estudiantes desarrollan por lo general, modelos o conceptualizaciones de lectura equivocados, los mismos, repercuten en la forma de efectuar la lectura de un texto. Hoy en día, existen varias instituciones dedicadas a la enseñanza de estrategias de comprensión lectora, porque precisamente sale a la luz el problema de la comprensión lectora de textos.

El estudiante, muchas veces suele aceptar como verdad absoluta los argumentos vertidos por el autor de un texto, por un lado; y por otro, retiene de manera arbitraria las proposiciones de un texto, obviando el significado de los mismos. Esto provoca el estancamiento de las ciencias que se aprenden. La percepción social, muestra la necesidad de construir nuevos conocimientos, que emerjan desde la realidad, que tengan como óptica, el mejorar la situación social del país, específicamente, mejorar la Administración de Justicia.

Como para todo problema, es necesario buscar una solución; y para ello, se debe partir de quien efectúa la lectura de un texto; y a quién va dirigido los beneficios de la misma, que no será sino el mismo lector. El bajo nivel de comprensión lectora de un texto por un lector, se encuentra concatenado con el modelo de lectura que desarrolla; la misma indudablemente repercute en la estrategias de comprensión lectora que utiliza (actividades o tareas que efectúa el lector para comprender un texto).

Las percepciones equivocadas sobre la lectura del estudiante que cursa la Asignatura de Filosofía del Derecho: (Como el aceptar como verdad absoluta el argumento vertido por el autor de un texto, por un lado; y por otro lado, la retención arbitraria de palabras como la memorización), repercute en el logro de los objetivos educativos de la Asignatura.

Estas conceptualizaciones equivocadas sobre la lectura, suelen darse a consecuencia de que los estudiantes van formando desde sus primeros cursos de formación escolar, falsas percepciones de comprensión lectora; las mismas son arraigadas como una especie de costumbre, desde sus primeros cursos de formación escolar.

El memorizar las proposiciones de los textos; o el aceptar como verdadero todo lo que se dice en los textos, provocan un inminente estancamiento en la formación del estudiante; que repercute en la ciencia que estudia, consecuentemente provoca un desfase en la relación escuela-sociedad: Satisfacción de necesidades sociales.

El tema que es presentado en este artículo, es apasionante, cuando la mirada se enmarca en mejorar la formación profesional de los estudiantes de Derecho, a partir de los valores socioculturales de la realidad boliviana; porque ello, es lo que se logrará cuando el estudiante construya nuevos conocimientos a partir de su realidad, de sus conocimientos previos, y de los que ya está escrito en los textos.

El conocer los tipos de modelos de lectura que desarrollan los lectores, los niveles de comprensión lectora que alcanzan, el objetivo educativo de la Asignatura de Filosofía del Derecho; si existe o no, influencia de los 
modelos de lectura en las estrategias de comprensión lectora, fueron los aportes teóricos y prácticos los que inspiraron la construcción de este estudio.

Buscando con ello, determinar la influencia de los modelos de lectura en las estrategias de comprensión lectora, para el desarrollo del modelo situacional de comprensión lectora, en los estudiantes de la Asignatura de Filosofía del Derecho, de la Carrera de Derecho, de la Universidad Mayor Real y Pontificia de San Francisco Xavier de Chuquisaca.

\section{MÉTODO}

Según Hernández Sampieri (2006) el diseño del presente trabajo de investigación científica, por su alcance es una investigación cuantitativa no experimental, transeccional correlacional. Toda vez que los datos recolectados se lo efectuó en un momento único y se describieron relaciones entre las variables de investigación: Modelos de Lectura y "Estrategias de Comprensión Lectora", en los estudiantes que cursan la Asignatura de Filosofía del Derecho, de la Carrera de Derecho, de la Universidad de San Francisco Xavier de Chuquisaca en la gestión.

La revisión histórica (Álvarez de Zayas, 1998) de información relativa a los modelos de lectura y estrategias de comprensión lectora, permitió encontrar modelos teóricos que mejor explican los fenómenos antes descritos: El modelo proposicional de comprensión de textos de Knitsch y Van Dijk, (1995) estudiada y amplificada por la Dra. Frida Díaz Barriga Arceo; y la clasificación de los modelos de lectura de los autores Schraw y Brunin (1996).

El método lógico (Álvarez de Zayas, 1998) permitió comprender y explicar la relación entre los niveles de comprensión lectora (microestructural, macroestructural y modelo de la situación) y la forma de alcanzarlos. También permitió identificar los componentes que intervienen en el proceso lector (lector, texto y contexto).

A partir de estos antecedentes, fue desarrollado modelo teórico que explique el fenómeno de la influencia de los modelos de lectura en las estrategias de comprensión lectora, en los estudiantes de la Asignatura de Filosofía del Derecho, a partir de la deducción y relación estructural funcional y holística de cuatro componentes (El lector, la actividad, el texto y el contexto).

El método de la medición (Álvarez de Zayas, 1998) permitió atribuir valores numéricos a los ítems de las variables "modelos de lectura" y "estrategias de comprensión lectora", lo que permitió efectuar una evaluación y representación adecuada acerca de la influencia de la primera variable con relación a la segunda, mediante el procedimiento estadístico SPSS.

La técnica de la encuesta, permitió recolectar información a partir de un cuestionario aplicado a los estudiantes que cursan la Asignatura de Filosofía del Derecho, acerca de los modelos de lectura y las estrategias de comprensión lectora que desarrollan.

La población estuvo conformada por los estudiantes inscritos en la Asignatura de Filosofía del Derecho, de la Carrera de Derecho, de la Universidad de San Francisco Xavier de Chuquisaca

\section{RESULTADOS Y DISCUSIÓN}

El modelo teórico que se presenta a continuación, es producto del análisis, deducción y relación de las teorías desarrolladas por los autores Schraw y Bruning (sobre conceptualizaciones de la lectura), Walter Knitsch y Teun Van Dijk (sobre el modelo proposicional de comprensión lectora) y Frida Díaz Barriga Arceo (sobre el modelo situacional de comprensión lectora). 
Es necesario acotar, que la actividad como una variante motivacional de los procesos de comprensión lectora, se incorpora como componente, dado que es un elemento que incorpora dos connotaciones. El primero, sirve de impulso al proceso de lectura, y el segundo que direcciona el mismo.

No es menos importante, los componentes que a simple observación pueden se deducidos en los procesos de comprensión lectora, tales como el lector (persona que efectúa la lectura y a quien va dirigida los beneficios de la misma), el texto (sobre la cual recae toda la actividad lectora, donde se encuentra plasmado todo el trabajo de su autor, dicho sea de paso, para algunos estudiosos el autor de un texto, también es considerado como componente de los procesos de comprensión lectora).

Finalmente el contexto (ámbito, lugar, sitio, donde se efectuó la lectura, misma influyó en la conducta y las actividades que desarrolló el lector). Convirtiéndose lo aseverado precedentemente, como un aporte del suscrito investigador, en aras de mejorar el desempeño de los estudiantes que cursan la Asignatura de Filosofía del Derecho.

\section{Enfoque del ModeloTeórico}

El presente modelo teórico, toma como postura el enfoque del paradigma cognitivo de procesamiento de la información propuesto por David Ausubel.

El lector, es el sujeto, la persona que desarrolla el proceso de comprensión lectora. En él se desarrolla niveles de comprensión lectora

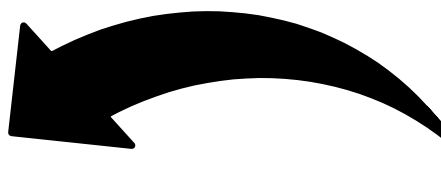

SUPERESTRUCTURA

Tiene conocimiento de la estructura formal del texto.

Efectúa una reflexión

Crítica acerca del Contenido del texto.

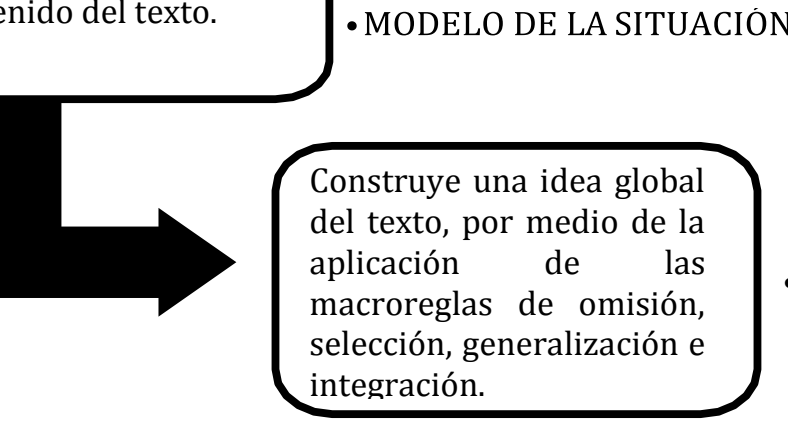

- MACROESTRUCTURAL.

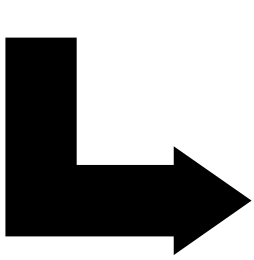

Decodifica el significado de las palabras y establece una relación lógica entre las mismas, el cual lo desarrolla casi de manera espontánea, desde su primer contacto con el texto. selección, generalización e integración.

- MICROESTRUCTURAL.

Figura 1. Características del componente lector del modelo teórico 
Relación entre Componentes.

\section{$\mathrm{C}_{1}=\mathrm{LECTOR}$}

$\mathrm{C}_{2}=$ ACTIVIDAD

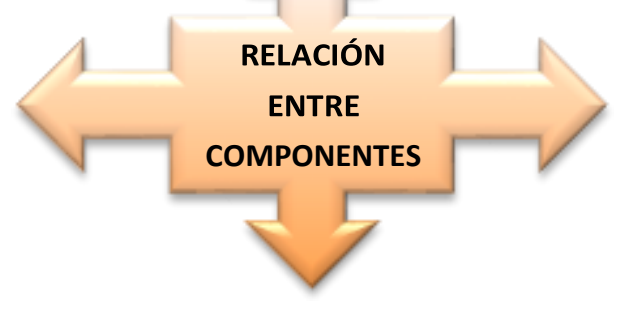

\section{$\mathrm{C}_{4}=$ CONTEXTO}

\section{$\mathrm{C}_{3}=$ TEXTO}

Figura 2. Relación entre los componentes del modelo teórico

\section{Relación Estructural Funcional}

El lector construye el significado del texto por medio de sus conocimientos previos, propósitos, intereses y motivaciones, entiende que un mismo texto puede interpretarse de manera diferente por distintos lectores, a pesar de las intenciones del autor o del contenido de un texto. Para arribar a esta situación es indudable que el mismo debe desarrollar estrategias de comprensión lectora, en base a modelos de transacción lectora.

\section{ACTIVIDAD}

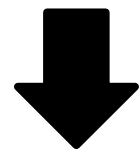

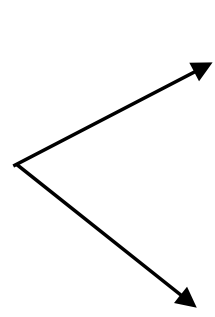

MODELO SITUACIONAL DE COMPRENSIÓN LECTORA

\section{CONTEXTO}

OBJETIVO EDUCATIVO DE LA ASIGNATURA: Uno de los problemas fundamentales que se evidencia actualmente en el ejercicio de la abogacía es que "no existe una auténtica teoría, una teoría práctica y crítica.

Figura 3. Relación estructural funcional 


\section{Relación Holística}

No se puede concebir un proceso lector, si obviamos siquiera uno de los componentes. $\mathrm{Si}$ bien por su importancia el componente Actividad, es el que permite desarrollar con éxito el proceso. El lector como agente a quien va dirigido el proceso, el texto, como la estructura formal de donde se extrae el conocimiento global de un contenido y el contexto, lugar donde se efectúa el proceso, son componentes esenciales para alcanzar el objetivo planteado.

\section{Estrategias de comprensión lectora en base al modelo transaccional de lectura, para el desarrollo del modelo situacional de comprensión lectora, en los estudiantes que cursan la Asignatura de Filosofía del Derecho}

\section{Justificación}

Como se ha podido establecer, los modelos de lectura influyen en la utilización de estrategias de comprensión lectora, en los estudiantes que cursan la Asignatura de Filosofía del Derecho, motivo por el cual se ha considerado importante realizar esta investigación con la presente alternativa propositiva para desarrollar el modelo situacional de comprensión lectora en los estudiantes que cursan la Asignatura de Filosofía delDerecho.

A partir del modelo transaccional de lectura, que mejor se adecúa al cumplimiento del objetivo citado, es que se ha elaborado estrategias de comprensión lectora, en el entendido que uno de los problemas fundamentales que se evidencia actualmente en el ejercicio de la abogacía es, que no existe una auténtica teoría, una teoría práctica y crítica. Y una teoría que rehúye la práctica o que acepta simplemente lo establecido, no pasa de ser falsa teoría.

Mucho nos quejamos de que nuestra legislación es copia fiel de legislaciones extranjeras, y que poco o nada hacemos para construir una normativa que esté acorde a nuestra realidad. Criticar es muy fácil, pero él no aportar ideas, es algo más penoso.

Para su mejor entendimiento de las estrategias de comprensión lectora que se propone, como medio para desarrollar el modelo situacional de comprensión lectora en los estudiantes que cursan la Asignatura de Filosofía del Derecho, se lo ha dividido en tres momentos: Antes, durante y después de la lectura de un texto.

\section{Objetivo}

Desarrollar, el modelo situacional de comprensión lectora, en los estudiantes que cursan la Asignatura de Filosofía del Derecho.

\section{Actividades}

De acuerdo a las necesidades y objetivos que se persiguen en la presente investigación (Desarrollar el modelo situacional de comprensión lectora), se ha establecido actividades a desarrollar durante los tres momentos del proceso de lectura: antes, durante y después de la lectura de un texto.

En el diseño de las actividades, se ha visto conveniente incorporar estrategias que permitan el control y monitoreo propio por parte del lector; son los denominados estrategias de monitoreo de la lectura, al igual según los intereses que se persiguen en el presente trabajo, se ha incorporado estrategias cuyas características permiten alcanzar los propósitos de la Asignatura de Filosofía del Derecho. 
El texto que utilizaremos para ejemplificar la aplicación de cada una de las estrategias propuestas, es un extracto del libro de Jaime Araujo Frías "Filosofía del Derecho. Una breve incitación a los abogados". FILOSOFÍA DEL DERECHO. UNA BREVE INCITACIÓN A LOS ABOGADOS

\section{IMPORTANCIA DE LA FILOSOFÍA DEL DERECHO PARA EL ABOGADO}

Sólo un abogado "con una disposición de apertura hacia el contenido de la filosofía del derecho podrá ser sensible a los reclamos que la sociedad le hace, para que con la entrega en el ejercicio de su profesión, recupere el lugar que un día tuvo el licenciado de derecho: respeto, confianza y veneración".115

Sostenemos que un abogado que quiera ejercitar su profesión al servicio de la sociedad con lucidez y honestidad116, no cabe duda que encuentra en la filosofía del derecho117 su mejor aliada. No con fines meramente teóricos, sino también prácticos. Pues la segunda presupone a la primera. Es sabido que no hay práctica inteligente118 sino está respaldada por una buena teoría119; es más, la práctica

115 Ruiz Rodríguez, Virgilio (2012). Filosofía del derecho. Toluca: Editorial Instituto Electoral del Estado de México, p. 234.

116 Pensamos que un abogado es ante todo un profesional del derecho. Una profesión es una actividad que lleva acabo alguien que es perito en una determinada área del saber humano. En nuestro caso concreto la actividad de resolver conflictos e incertidumbres jurídicas a través de normas jurídicas. En efecto, lo que da sentido a la profesión de la abogacía es el bien que ofrece y que ninguna otra profesión puede dar: posibilitar la justicia a través de normas. Y el abogado que actúa así, créase o no, parafraseando al Nobel en economía Amartya Sen, es un bien público y no un peligro para la sociedad.

117 Sostenemos que la filosofía del derecho es una rama de la filosofía que se ocupa de la reflexión crítica y ordenada sobre la naturaleza del derecho, sobre la justificación de los saberes que aparecen en el mundo jurídico y, sobre cómo y por qué se deben orientar la práctica jurídica hacia la posibilitación de la justicia.

118 Definimos la inteligencia como la capacidad para enfrentar y resolver problemas de manera creativa.

119 Una teoría es un edificio conceptual formado por una colección organizada de nociones y proposiciones que codifica información acerca de cierto tipo de sistemas, fenómenos o procesos y típicamente sirve para dar explicaciones, hacer predicciones y resolver problemas de manera creativa. Cfr. Mosterín J. y Torretti R. (2002). Diccionario de lógica y filosofía de la ciencia. Madrid: Alianza, p. 556. 


\section{JAIME ARAUJO FRIAS}

jurídica siempre debe ser edificada sobre la buena teoría; y la filosofía jurídica, por su objeto mismo, "no solo se ocupa de problemas teóricos más o menos especulativos, sino que se ve también obligada a entrar en contacto con la realidad social en uno de sus aspectos más inquietantes: el de la regulación del comportamiento humano a través de la coacción".120

En tal sentido, la aseveración de Manuel Atienza expuesta como pretexto para iniciar el presente apartado se justifica, porque pensamos con él que uno de los problemas fundamentales que se evidencia actualmente en el ejercicio de la abogacía es que "no existe una auténtica teoría, una teoría práctica y crítica. Y una teoría que rehúye la práctica o que acepta simplemente lo establecido, no pasa de ser falsa teoría."121 Pues, la teoría no es otra cosa que una estrategia lúcida y eficaz para la consecución de fines concretos y no un decorado para solapar la ineficiencia. Por ello, sugerimos la implementación del curso de filosofía en las facultades de derecho y en las que ya la tienen darle mayor importancia (Araujo Frías, 2015).

120 Garzón Valdés, E. y Laporta, J. (2013). El derecho y la Justicia. Enciclopedia Iberoamericana de filosofía. Madrid: Trotta, p. 11.

121 Atienza, Manuel (2008). Ideas para una filosofía del derecho. Una propuesta para el mundo Latino. Lima: Universidad Inca Garcilaso de la Vega, p. 23.

Figura 3. Extracto del libro "filosofía del derecho". Una breve incitación a los abogados

\section{Estrategias de comprensión lectora previas a la lectura}

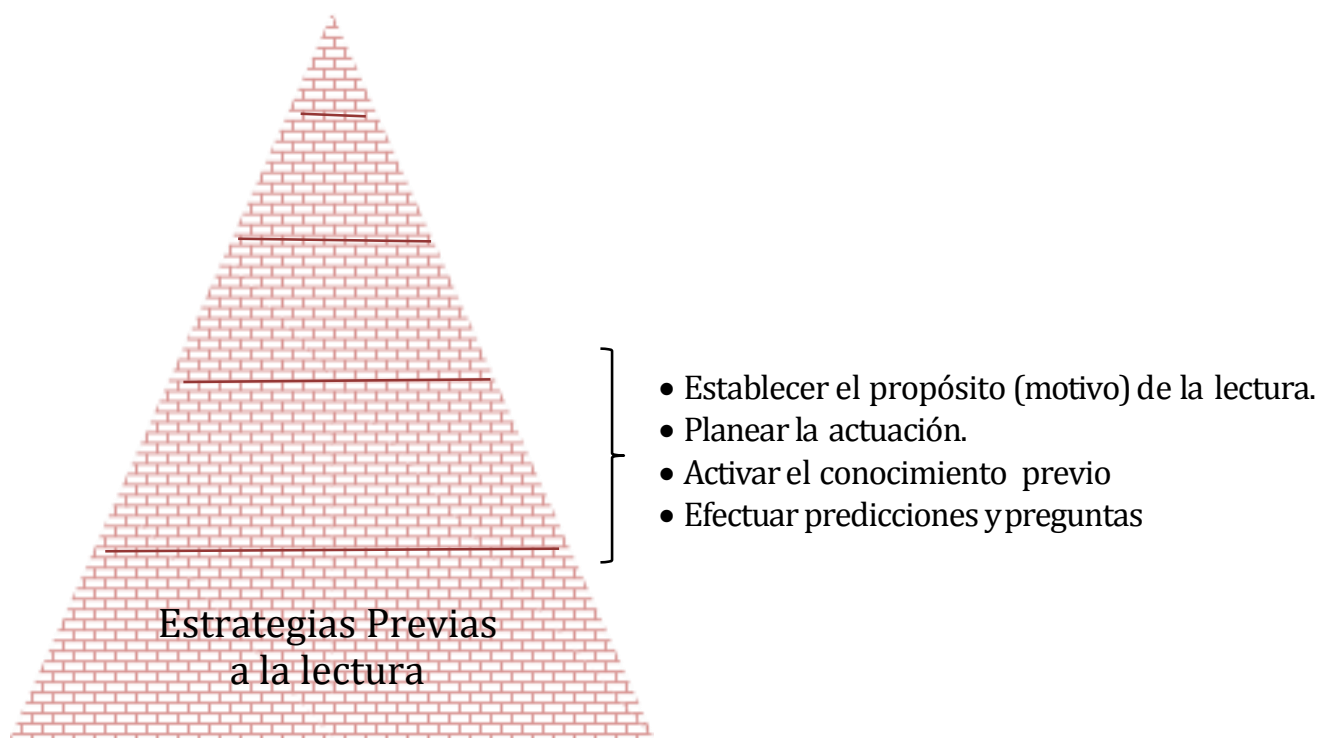

Figura 4. Estrategias de comprensión lectora previas a la lectura. 


\section{Establecer el propósito de la lectura}

El establecimiento del propósito (motivo) de la lectura (el interés que nos mueve para efectuar la lectura) se encuentra delimitado por el objeto de estudio de la Asignatura: "La filosofía del derecho es una rama de la filosofía que se ocupa de la reflexión crítica y ordenada sobre la naturaleza del derecho, sobre la justificación de los saberes que aparecen en el mundo jurídico y, sobre cómo y por qué se deben orientar la práctica jurídica hacia la posibilitación de la justicia". En base a este propósito u objetivo de la ciencia que se estudia, se debe establecer el objetivo de la lectura.

Ejemplo:

Asignatura: $\quad$ Filosofía del Derecho.

Tema: Importancia de la filosofía del derecho para el abogado.

Objetivo de la lectura del texto: Identificar los factores positivos que hacen necesario el estudio de la filosofía del derecho una ciencia imprescindible en la formación del abogado.

Figura 5. Construcción del objetivo de la lectura de un texto.

Resultado. Tener plenamente establecido el propósito de la lectura, permitirá al estudiante direccionar el proceso de lectura, por medio de una planeación adecuada y predecir los resultados esperados.

\section{Planificar la actuación}

El establecimiento del propósito de la lectura, permitirá delinear las estrategias que se utilizarán, desde un inicio, durante y después de la lectura, tomando en cuenta también la estructura formal del texto y la magnitud del contenido.

Ejemplo:

Tema: $\quad$ Importancia de la filosofía del derecho para el abogado.

Objetivo de la lectura del texto: Identificar los factores positivos que hacen necesario el estudio de la filosofía del derecho una ciencia imprescindible en la formación del abogado.

Estructura formal del texto: Expositivo.

Magnitud del texto: 2 paginas.

Planificación: El texto, es expositivo de dos páginas, el tiempo que nos tomará efectuar la lectura no es extenso.

Figura 6. Planificación de la actuación de un proceso de comprensión lectora. 
Resultado. Permitirá conocer el tiempo requerido para efectuar la lectura y la forma de aplicación de las estrategias de comprensión lectora.

\section{Activación del conocimiento previo}

Esta estrategia es considerada elemental por el enfoque y la finalidad del presente trabajo. Una actividad sumamente efectiva para activar el conocimiento previo es, la denominada actividad focal introductoria. Esta actividad consiste que el lector busque fuentes que vayan en contradicción a lo aseverado en el texto.

\section{Ejemplo:}

Tema: Importancia de la filosofía del derecho para el abogado

Objetivo de la lectura del texto: Identificar los factores positivos que hacen necesario el estudio de la filosofía del derecho una ciencia imprescindible en la formación del abogado.

Actividad focal introductoria: Encontrar argumentos: "Que la filosofía del derecho, no sirve para nada".

Magnitud del texto: 2 paginas.

Figura 7. Construcción del conocimiento previo.

Resultado. Esta manifestación, provocará ser el foco de atención y discusión en el lector (motivación). El mismo necesariamente tendrá que refutar o aprobar dicha aseveración, cuando culmine de efectuar la lectura del texto.

\section{Efectuar predicciones y preguntas}

Esta estrategia sirve para proponer un contexto, también facilita la activación y el uso del conocimiento previo (ya sea el relacionado con el tópico del texto o el conocimiento sobre la organización estructural del texto). En esta actividad el lector debe efectuar un somero repaso del texto (observando el índice, los títulos y subtítulos...), para saber el tiempo y construir preguntas a responder durante la lectura del texto. 


\section{Ejemplo:}

Tema: Importancia de la filosofía del derecho para el abogado

Objetivo de la lectura del texto: Identificar los factores positivos que hacen necesario el estudio de la filosofía del derecho una ciencia imprescindible en la formación del abogado.

- $\quad$ El texto no es extenso, por la forma es un texto expositivo.

- Formularse preguntas: ¿Cuáles son los factores positivos que hacen del estudio de la filosofía del derecho, una ciencia importante en la formación delabogado?

Magnitud del texto: 2 paginas.

Figura 8. Elaboración de predicciones y preguntas.

Resultado. Predecir, es adelantarse a los hechos, el mismo permitirá materializar y dirigir el proceso de lectura.

\section{Estrategias de comprensión lectora previas a la lectura}

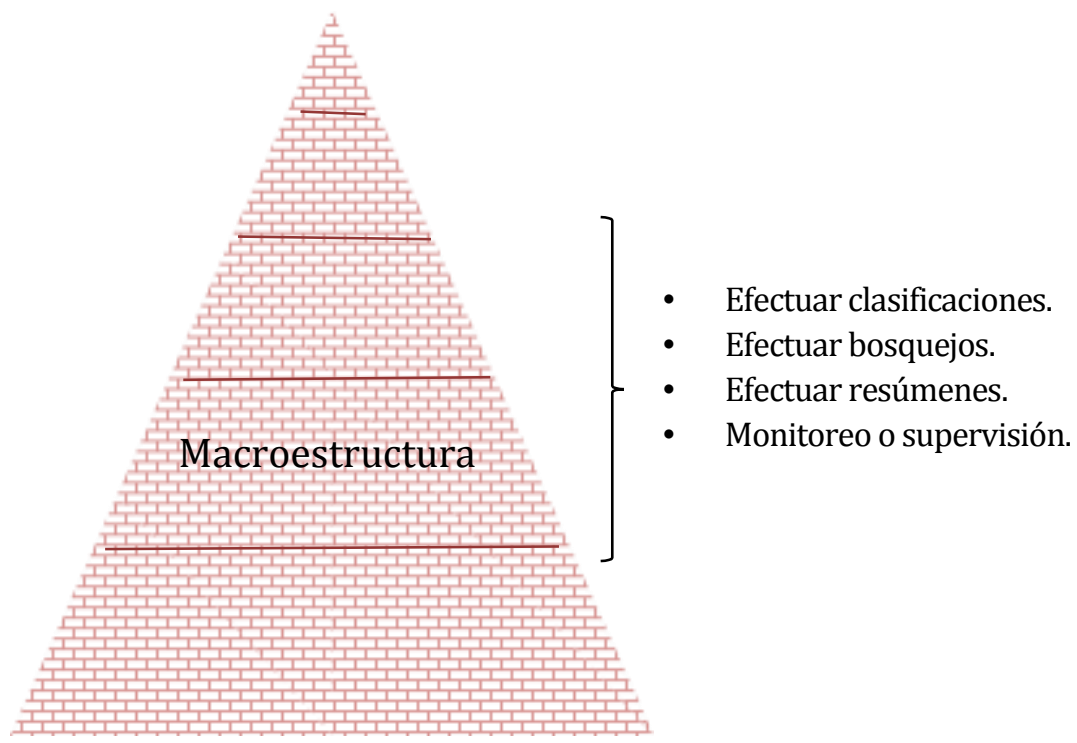

Figura 9. Estrategias de comprensión lectora durante la lectura

\section{Efectuar clasificaciones}

Al momento de efectuar la lectura de un texto, es necesario categorizar personas, objetos, lugares, etc... 


\section{Ejemplo:}

Tema: Importancia de la filosofía del derecho para el abogado

Objetivo de la lectura del texto: Identificar los factores positivos que hacen necesario el estudio de la filosofía del derecho una ciencia imprescindible en la formación del abogado.

Abogado al servicio de la sociedad con lucidez y honestidad Filosofía del Derecho.

Realidad social

Manuel Atienza

Teoría práctica y crítica

Figura 10. Categorización de los objetos que forman parte del texto.

Resultado. El efectuar una categorización de los objetos que forman parte del texto, permitirá una jerarquización de las proposiciones o de las ideas del texto.

\section{Efectuar bosquejos}

Es necesario reproducir de manera esquemática el contenido de un texto. El mapa de contenidos por perseguir un objetivo, a diferencia del mapa conceptual, es el más recomendable elaborar. 


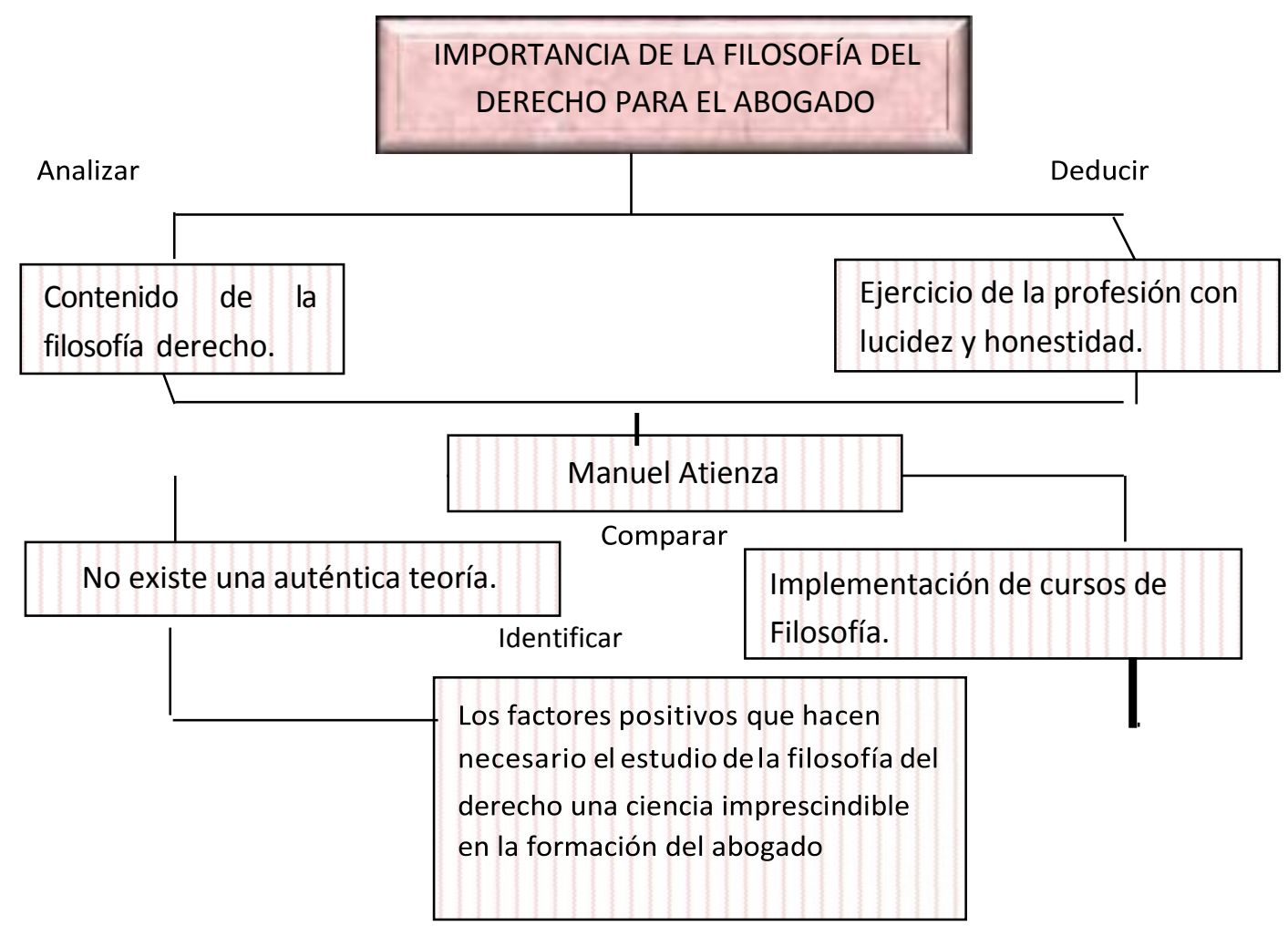

Figura 11. Mapa de contenidos.

Resultado. El esquematizar el contenido de un texto, permitirá obtener un entendimiento global de la lectura de un texto.

\section{Efectuar resúmenes}

Condensar el texto, implica efectuar un resumen acumulativo (resumir por partes e irlas acumulando hasta acabar con el texto), de este modo puede tener un resumen en forma cronológica del contenido del texto.

\section{Ejemplo:}

Tema: Importancia de la filosofía del derecho para el abogado

Objetivo de la lectura del texto: Identificar los factores positivos que hacen necesario el estudio de la filosofía del derecho una ciencia imprescindible en la formación del abogado.

Resumen. La Filosofía del Derecho, sirve para ejercitar la profesión del abogado con lucidez y honestidad, ya que uno de los problemas en el ejercicio de la abogacía es que no existe una autentica teoría práctica, por ello que se sugiere la implementación del curso de filosofía.

Figura 12. Elaboración de resúmenes

Resultados. Permite una descripción sintética del contenido de un texto. 


\section{Monitoreo o supervisión}

Esta actividad autorreguladora se ejecuta en función del propósito y del plan previamente especificado. Para ello el lector debe sentir que está comprendiendo, debe saber si entiende o no lo suficiente y resolver los distintos problemas u obstáculos que vayan apareciendo durante el proceso de comprensión lectora.

\section{Ejemplo:}

Tema: Importancia del pensamiento crítico para el abogado.

Objetivo general: El estudiante desarrolle capacidades de reflexión crítica y ordenada sobre la naturaleza de la ciencia del derecho.

Objetivo específico: El estudiante desarrolle una actitud crítica frente a los problemas sociales de justicia.

Actividad autorreguladora: Durante el transcurso de la lectura preguntarse si realmente estoy comprendiendo el texto. Y si no comprendo algún término recurrir a diccionarios especializados, o finalmente consultar a los docentes.

Figura 13. Monitoreo y supervisión de la lectura.

Resultado. Esta actividad permitirá al lector direccionar la lectura. Permite desarrollar un análisis interno en el lector; es decir, si está cumpliendo con los objetivos propuestos momentos antes de iniciar la lectura.

\section{Estrategias de comprensión lectora previas a la lectura}

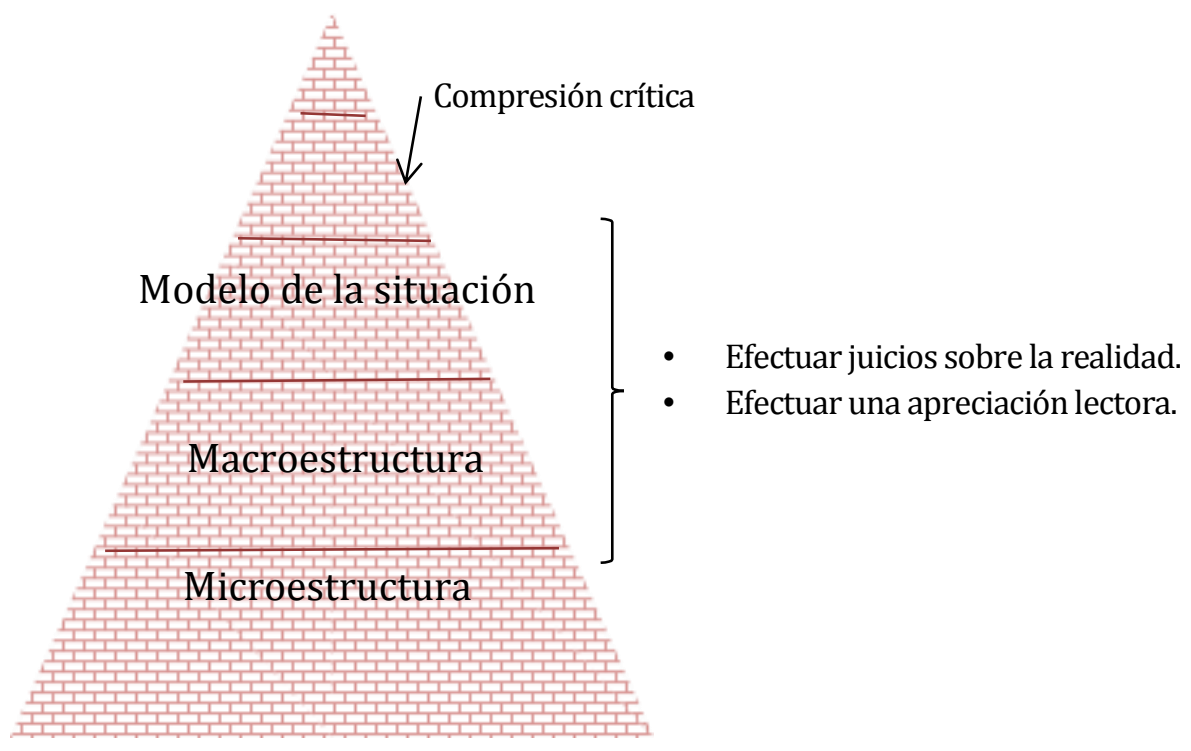

Figura 14. Estrategias de comprensión lectora después de la lectura 


\section{Efectuar juicios sobre la realidad}

El lector necesita establecer una relación entre la información del texto y los conocimientos que ha obtenido de otras fuentes, y evaluar las afirmaciones del texto contrastándolas con su propio conocimiento del mundo.

\section{Ejemplo:}

Tema: Importancia de la filosofía del derecho para el abogado

Juicio sobre la realidad. La administración de justicia, se encuentra atravesando por un momento crítico, toda vez que la legislación no responde a la situación social de nuestro medio.

Figura 15. Elaboración de juicios sobre la realidad.

Resultados. Permite la reflexión sobre el contenido del texto.

\section{Efectuar una apreciación lectora}

El lector debe efectuar las relaciones espaciales y temporales, ambigüedades léxicas y relaciones entre los elementos de las proposiciones del texto.

\section{Ejemplo:}

Tema: Importancia de la filosofía del derecho para el abogado

Apreciación lectora. El texto es corto en su extensión, de tipo expositivo, toma en cuenta fuentes de varios autores, pero a su vez efectúa un análisis y apreciación propia del texto.

Figura 16. Apreciación lectora

Resultados. Permite realizar una reflexión sobre la forma del texto, ya que se requiere un distanciamiento por parte del lector, una consideración objetiva de éste y una evaluación crítica y una apreciación del impacto de ciertas características textuales como la ironía, el humor, el doble sentido, etc.

\section{Validación Teórica de la Propuesta}

\section{Aplicación del Cuestionario Delphi para el criterio de expertos}

Por último se aplicó el cuestionario Delphi para de recabar información acerca de la factibilidad de la propuesta: "Estrategias de comprensión lectora en base al modelo transaccional de lectura, para el desarrollo del modelo situacional de comprensión lectora, en los estudiantes que cursan la Asignatura de Filosofía del Derecho". En donde se demostró la factibilidad del estudio desarrollado.

\section{CONCLUSIONES}

Se estableció a partir de los autores Shraw y Bruning, que son tres los modelos de lectura que desarrollan los lectores (de translación, de transmisión y de transacción de lectura). Según los autores Knitsch y Van 
Dijk, los niveles por los cuales atraviesa el lector en el proceso de comprensión lectora de un texto son: Microestructural (codificación de las proposiciones del contenido de un texto), macroestructural (construcción de la idea principal de un texto), a estos dos niveles, Frida Díaz Barriga incorpora el modelo situacional de comprensión lectora (mundo análogo a los niveles descritos, en el que intervienen las ideas del texto y los conocimientos previos que tiene el lector). Por otra parte, según Frida Díaz Barriga Arceo, que el proceso de comprensión lectora de textos, es una actividad constructiva, compleja y estratégica.

Como lo manifestado Frida Díaz Barriga Arceo, para alcanzar los niveles de comprensión lectora, el lector debe realizar actividades de microprocesamiento, macroprocesamiento y modelo de la situación. Se determinó que los estudiantes que cursan la Asignatura de Filosofía del Derecho, en su mayoría desarrollan un modelo de transmisión de lectura, este porcentaje no pasa de la mitad de los encuestados. Además, que los estudiantes que cursan la Asignatura de Filosofía del Derecho, desarrollan actividades de macroprocesamiento para comprender la lectura de un texto. A partir de la comprobación de la hipótesis planteada mediante la prueba estadística Chi cuadrada, se ha determinado que los modelos de lectura influyen en la utilización de estrategias de comprensión lectora en los estudiantes que cursan la Asignatura de Filosofía del Derecho.

Se ha identificó dentro la influencia de los modelos de lectura en la utilización de estrategias de comprensión lectora en los estudiantes de la Asignatura de Filosofía del Derecho, cuatro componentes: El lector, el texto, el contexto, y la ACTIVIDAD. La relación teórica y gráfica de los cuatro componentes permitió fundamentar su relación interna y fundamentar de manera científica su proceso.

A partir de la comprobación de la influencia de los modelos de lectura en la utilización de estrategias de comprensión lectora, en los estudiantes que cursan la Asignatura de Filosofía del Derecho, se diseñó estrategias de comprensión lectora edificadas a partir del modelo de transacción de lectura.

Se obtuvo la validación teórica y práctica de la propuesta pedagógica, obteniendo como resultado como muy adecuado las estrategias de comprensión lectora propuestas en el presente trabajo de investigación científica.

\section{REFERENCIAS}

Ausubel, D. P. (1982). A aprendizagem significativa: a teoría de David Ausubel. São Paulo: Moraes, 198.

Álvarez de Zayas, C. M. (1998). Metodología de la Investigación Científica. Cochabamba: IPCE-ECEM.

Araujo Frías, J. (2015). Filosofía del Derecho, una breve incitación a los abogados. ChileArgentina: Abierta.

Atienza, M. (2008). Ideas para una Filosofía del Derecho. Una propuesta para el mundo Latino. Lima: Universidad Inca Garcilazo de la Vega

Barriga Arceo, F. D. (2002). Estrategias Docentes para un aprendizaje significativo. Una interpretación constructivísta. México: Cámara Nacional de la Industria Editorial Mexicana

Comprensión lectora: historia y componentes del proceso lector. (Noviembre de 2014). Obtenido de

http://publicacionesdidacticas.com/hemerot eca/articulo/052032/articulo-pdf

Estévez, E. E. (1990). Estrategias cognitivas para la comprensión de textos en educación superior. 10

Hernández Sampieri, R. F. (2006). Metodología de la Investigación (Cuarta ed.). México: Talleres de Infagon Web.

Kintsch, W., y Van Dijk, T. A. (1978). Toward a model of text comprehension and 
production. Psychological review, 85(5), 363.

Ruiz Rodríguez, Virgilio (2012). Filosofía del derecho. Toluca: Editorial Instituto Electoral del Estado de México, p. 234

Schraw, G. y Bruning, R. (1996). Modelos implícitos de lectura de los lectores.
Reading Research Quarterly 31 (3), 290305

Van Dijk, TA (1995). Sobre macroestructuras, modelos mentales y otros inventos: una breve historia personal de la teoría de Kintsch-van Dijk. Comprensión del discurso: Ensayos en honor de Walter Kintsch, 383-410 